\title{
Hemorrhagic cholecystitis: report of a case
}

\begin{abstract}
Jung-Nam Kwon
Department of Surgery, Wonkwang University Sanbon Hospital, Wonkwang University School of Medicine, Gunpo, Korea

Hemorrhagic cholecystitis is an uncommon cause of abdominal pain that can be fatal. We report a case of hemorrhagic cholecystitis in a 75-year-old male taking an anticoagulant. The patient was brought to the hospital with uncontrolled right upper quadrant abdominal pain. On computed tomography, mild gallbladder wall thickening and high density with gallstones in the gallbladder suggested acute calculous cholecystitis or hemorrhagic cholecystitis. An urgent laparoscopic cholecystectomy was performed that revealed a gallbladder filled with large blood clots and two black stones. Patients who develop hemorrhagic complications were often receiving anticoagulation therapy or had pathologic coagulopathy. An early diagnosis of this potentially fatal condition is important to facilitate urgent surgical treatment. (Korean J Hepatobiliary Pancreat Surg 2012;16:120-122)
\end{abstract}

Key Words: Hemorrhagic; Cholecystitis; Anticoagulation; Urgent; Laparoscopic cholecystectomy

\section{INTRODUCTION}

Hemorrhagic cholecystitis is a rare complication of acute cholecystitis. Hemorrhagic cholecystitis is hard to detect as its symptoms are easily confused with more common diagnoses. A hemorrhage within the gallbladder lumen may occur for a variety of reasons, including obstructive cholecystitis, trauma, percutaneous interventions, biliary neoplasms, biliary parasites, or bleeding disorders. ${ }^{1}$ A computed tomography (CT) can help diagnose and demonstrate the characteristic findings of wall thickening of the distended gallbladder and the heterogenous materials inside. Here, we report the case of a patient receiving anticoagulation therapy with hemorrhagic cholecystitis.

\section{CASE}

A 75-year-old male was admitted with a 15-hour history of fever and right upper quadrant abdominal pain. His pain was not controlled by a pain killer and he was irritable. He was on Warfarin $2 \mathrm{mg}$ for atrial fibrillation for 5 years without complication. His past medical history included hypertension, smoking but no diabetes. A phys- ical examination revealed direct tenderness and rebound tenderness in the right upper abdominal quadrant with rigidity. His white cell count was $14.1 \times 10^{3} / \mathrm{ul}$ (neutrophils $86.1 \%$ ) and erythrocyte sedimentation rate (ESR) 17 $\mathrm{mm} / \mathrm{hr}$. The results of chemistry studies included a total bilirubin of $1.77 \mathrm{mg} / \mathrm{dl}$ with direct bilirubin $0.64 \mathrm{mg} / \mathrm{dl}$, aspartate aminotransferase of $640 \mathrm{IU} / \mathrm{L}$, alanine aminotransferase of $167 \mathrm{IU} / \mathrm{L}$, gamma-glutamyltransferase of $375 \mathrm{IU} / \mathrm{L}$. At presentation, his prothrombin time (PT) was elevated measuring $28.4 \mathrm{sec}$, INR was elevated measuring 2.4, but aPTT was not elevated. The CT scan of the abdomen with intravenous contrast showed few gallstones with mild gallbladder wall thickening, high density in gallbladder and bile duct. A CT scan suggested sludge, a stone or tumor obstructing the distal common bile duct combined with acute cholecystitis that was unlikely hemobilia or hemorrhagic cholecystitis (Fig. 1).

Based on the patient's symptoms, signs and CT findings, the patient underwent an urgent laparoscopic cholecystectomy. A distended edematous gallbladder was found and filled with dark blood clots (Fig. 2). The patient underwent an uneventful laparoscopic cholecystectomy and his pain was controlled. The next day, his bilirubin

Received: June 18, 2012; Revised: July 25, 2012; Accepted: July 26, 2012

Corresponding author: Jung-Nam Kwon

Department of Surgery, Wonkwang University Sanbon Hospital, Wonkwang University School of Medicine, 1142, Sanbon-dong, Gunpo 435-040, Korea

Tel: +82-31-390-2218, Fax: +82-31-390-2244, E-mail: jnamii@hanmail.net 

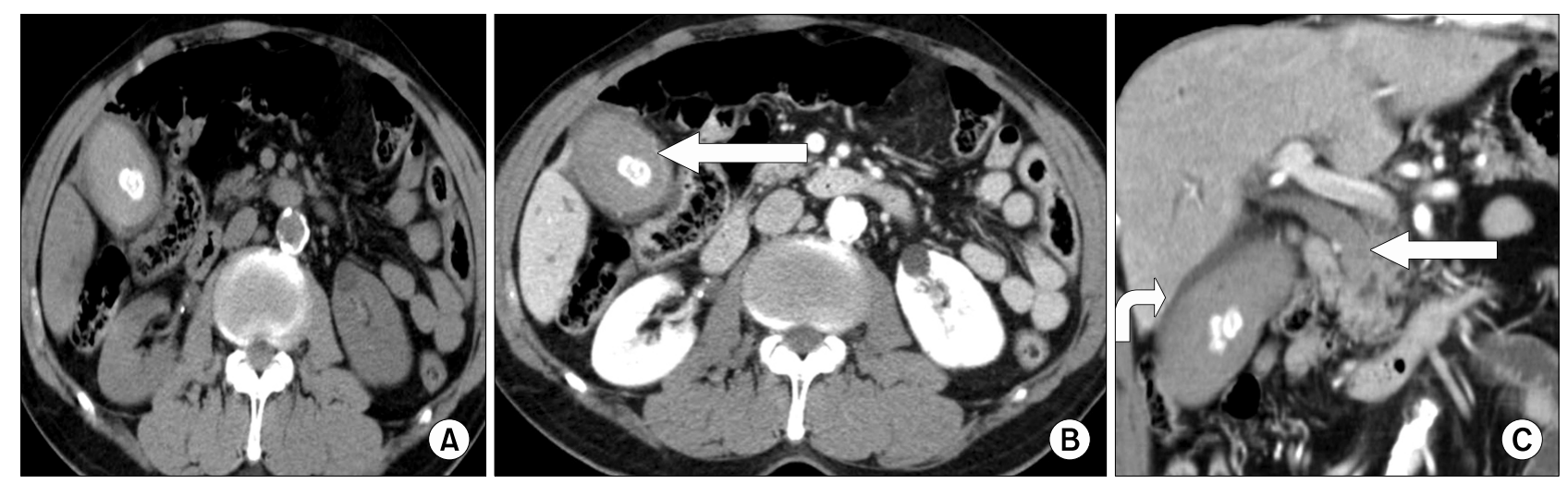

Fig. 1. Preoperative CT scan. (A) A non-contrast CT revealed highly attenuated materials in the gallbladder and extra-hyperdense gallstones. (B) An enhanced CT image obtained in the arterial phase demonstrated streaks-like enhancement along the gallbladder wall (arrow). (C) The coronal arterial phase CT showed that the gallbladder (curved arrow) and common bile duct (CBD) (straight arrow) were distended and contained high density fluid. The gallbladder fluid was confirmed as a large amount blood clots and CBD dilatation due to sludge and tiny stones.

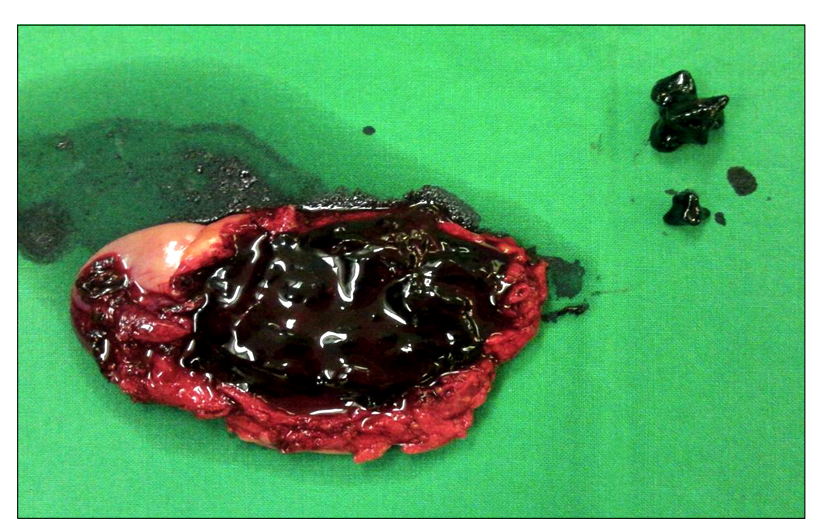

Fig. 2. A gross photograph of the gallbladder specimen (about $8.4 \times 4.1 \mathrm{~cm}$ ) with wall thickening is filled with the remaining intraluminal organized blood clots (total about $50 \mathrm{~g}$ ) and two black stones.

was elevated (total bilirubin of $7.14 \mathrm{mg} / \mathrm{dl}$; direct bilirubin 2.64). The $3^{\text {rd }}$ postoperative day endoscopic retrograde cholangiopancreatography (ERCP) was performed, which showed a dilated biliary tract with sludge and stones but there was no evidence of hemobilia. Sludge and small stones were removed by an endoscopic sphincterotomy (EST) and endoscopic naso-biliary drainage (ENBD) was performed then removed 2 days later.

A histologic examination of the gallbladder showed acute erosion and a focal gangrenous lesion however it was not clear whether it was from a transmural bleeding. His symptoms and laboratory findings had all improved and anticoagulation therapy was uneventfully reinitiated. He was discharged 9 days after admission. At the fol- low-up examination 3 months after surgery, he was asymptomatic and doing well.

\section{DISCUSSION}

Hemorrhage within the gallbladder lumen is a rare cause of abdominal pain that can present due to trauma, malignancy, and bleeding diathesis, such as renal failure, cirrhosis, and anticoagulation. Hemorrhagic cholecystitis is easily overlooked since its symptoms mimic those of common hepatobiliary diseases with right upper quadrant abdominal pain, liver function impairment, leukocytosis and positive Murphy's sign. ${ }^{2}$

Pathologically, there is a transmural wall inflammation which causes infarction and erosion of the mucosa, which in turn may cause hemorrhage into the gallbladder lumen. A CT may demonstrate density fluid with or without layering, which may mimic sludge, such as "milk of calcium". ${ }^{3}$ Pandya and O'Malley ${ }^{4}$ emphasized the value of the arterial phase of a contrast-enhanced CT, which may demonstrate active extravasation of the contrast into the lumen of the gallbladder. But in this case CT scan showed hyperdense contents within the gallbladder and may show thread-like steaks of contrast escaping along the gallbladder wall (Fig. 1).

CBD obstruction with subsequent jaundice and biliary colic may be also be caused due to a blood clot. $^{5}$ Hyperdense material is also present within the CBD in this case, but, the ERCP showed a dilated biliary tract 
with sludge and stones, but there was no evidence of hemobilia.

Hemorrhagic cholecystitis has been reported in both the presence and absence of cholelithiasis and is associated with high morbidity and mortality rates, especially when complicated with gallbladder perforation and massive hemorrhage. ${ }^{3,6-8}$ Regardless of the cause, cholecystitis required surgery in most cases. In patients who are not surgical candidates, a cholecystotomy is an option. There was a report of hemorrhagic cholecystitis treated with a cholecystostomy and urokinase therapy. ${ }^{9}$

But, Pandya and $\mathrm{O}^{\prime}$ Malley ${ }^{4}$ reported a case complicated by anticoagulant therapy that underwent an urgent cholecystostomy and unfortunately the hyperdense contents increased within the gallbladder and CBD in follow-up CT. Treatment for hemorrhagic cholecystitis during anticoagulant therapy needs an urgent cholecystectomy which is necessary to prevent more serious complications.

In summary, hemorrhagic cholecystitis is rare but its complications are not rare. They are often fatal. A high index of suspicion is required, especially if there are predisposing factors like anticoagulant therapy. Early diagnosis of this potentially fatal complication is important to facilitate urgent surgical management.

\section{ACKNOWLEDGEMENTS}

This paper was supported by Wonkwang University in 2010 .

\section{REFERENCES}

1. Morris DS, Porterfield JR, Sawyer MD. Hemorrhagic cholecystitis in an elderly patient taking aspirin and cilostazol. Case Rep Gastroenterol 2008;2:203-207.

2. Lai YC, Tarng DC. Hemorrhagic acalculous cholecystitis: an unusual location of uremic bleeding. J Chin Med Assoc 2009; 72:484-487.

3. Tavernaraki K, Sykara A, Tavernaraki E, et al. Massive intraperitoneal bleeding due to hemorrhagic cholecystitis and gallbladder rupture: CT findings. Abdom Imaging 2011;36:565-568.

4. Pandya R, O'Malley C. Hemorrhagic cholecystitis as a complication of anticoagulant therapy: role of $\mathrm{CT}$ in its diagnosis. Abdom Imaging 2008;33:652-653.

5. Parekh J, Corvera CU. Hemorrhagic cholecystitis. Arch Surg 2010;145:202-204.

6. Jenkins M, Golding RH, Cooperberg PL. Sonography and computed tomography of hemorrhagic cholecystitis. AJR Am J Roentgenol 1983;140:1197-1198.

7. Nural MS, Bakan S, Bayrak IK, et al. A rare complication of acute cholecystitis: transhepatic perforation associated with massive intraperitoneal hemorrhage. Emerg Radiol 2007;14:439-441.

8. Heo TY, An YY, Lee JH, et al. A case of spontaneous hemorrhagic cholecystitis without gallstone. Korean J Gastroenterol 2010;56:260-263.

9. Stempel LR, Vogelzang RL. Hemorrhagic cholecystitis with hemobilia: treatment with percutaneous cholecystostomy and transcatheter urokinase. J Vasc Interv Radiol 1993;4:377-380. 